\title{
A fully equilibrated microsphere model with damage for rubberlike materials
}

\author{
J. Diani, P. Le Tallec \\ Laboratoire de Mécanique des Solides, UMR 7649, Ecole Polytechnique, 91128 Palaiseau, \\ France
}

\begin{abstract}
A non-affine microsphere model for rubberlike materials is proposed, based on a local minimization of the network free energy under a maximal advance path constraint. It accounts for any chain weight distribution and for damage such as Mullins softening observed in filled rubber materials. The non-affine equal-force model is compared to the common affine model and a hybrid equal-force model from the literature, when considering the isotropic hyperelastic behavior without damage of rubber materials presenting chains of various lengths. The non-affine model shows an improved deformability compared to the affine model limited by the maximal extension of the shorter chains and a significantly softer behavior. Possible damage is introduced by increasing the chain lengths according to the submitted maximal chain traction force. Each chain are impacted independently resulting in a directional softening that introduces the evolution of the stress-free configuration that needs to be assessed over the loadings. The model was successfully tested on the cyclic uniaxial tension stretch-stress responses of carbon-black filled styrene butadiene rubbers that were well fitted with three parameters only.

Keywords: rubber material, constitutive behavior, finite strain, anisotropic material, Mullins softening
\end{abstract}

\section{Introduction}

Rubberlike materials are made of long polymer chains crosslinked into physical networks. Large strains are attained thanks to the uncoiling of chains while elasticity is achieved by the crosslinkings. From very early on, micromechanically-based models have been developed to reproduce the en- 
tropic elasticity of these materials. Assuming an ideal network, the change of its entropy is defined as the sum over the chains of their entropy changes. Based on the statistics of polymeric chain configurations, Gaussian or Langevin chain force-elongation relations have been defined (Kuhn and Grün, 1942). Dealing with isotropic materials, chains are randomly oriented in space, which is conveniently represented by a microsphere, the chain end-toend vectors going from the center to the surface of the sphere, as proposed by Treloar and Riding (1979) in their full-network model. The change of scale from the material macroscopic scale at which the mechanical loading is applied to the microscopic chain scale, may be specified by various assumptions, the most common being the affine one. For the latter, each chain is assumed to move freely without constraint of its environnement, and the macroscopic deformation gradient is directly applied to every chain end-to-end vector.

Non-affine models have been proposed to account for more realistic chain deformations since long polymer chains are entangled and are assumed to move within virtual tubes (McLeish, 2002) that create topological constraints. In a seminal work, Miehe et al. (2004) defined a microsphere model with non-affine deformation, adding a stretch-fluctuation field to the affine chain stretch field. The solution satisfies to the kinematic compatibility conditions while minimizing the energy state. Following a similar path, Tkachuk and Linder (2012) proposed an efficient minimum energy based model thanks to the description of chain paths for networks of functionalities three and four. Finally, Verron and Gros (2017) proposed an hybrid equal-force model, assuming equal-force for chains that are aligned, rather than equal-stretch like for the affine model, with the constraint of collinearity of the deformed end-to-end chain vectors with the affine deformed end-to-end vectors.

When used for industrial applications, rubberlike materials are usually filled with nano-size fillers such as carbon-black or silica. The reinforcing particles benefit to wear and failure resistances significantly, without sacrificing the large strain elasticity. Nonetheless, such filled rubbers exhibit a substantial softening upon first stretch, known as Mullins softening (Mullins, 1969). At the macroscopic scale, the Mullins softening was shown to be a directional damage reactivating when exceeding the maximum load already applied (Merckel et al., 2012), probably due to debonding an unraveling of the bounded layer of polymer chains at the gum/particle interface (Diaz et al., 2014). The damage and induced anisotropy resulting from the Mullins softening, have been well reproduced by introducing directional damage in affine microsphere models (Göktepe and Miehe; 2005, Diani et al., 2006), but 
have never been taken into account within a non-affine three-dimensional microsphere model framework. The main difficulty stands in the evolution of the stress-free configuration that is damage dependent. Such a difficulty was not encountered by Rastak and Linder (2018) when they introduced strain-induced crystallization in the Tkachuk and Linder (2012) model. In the current contribution, a non-affine microsphere model for rubberlike materials with damage is defined based on the convenient kinematic framework defined in Tkachuk and Linder (2012), and extended herein to a general thermodynamic environment. The Mullins softening is introduced as directional evolution of the polymer chain lengths, since increasing the chain lengths loosen the polymer network rendering it softer.

The paper is organized as follows. In section 2, the model of Tkachuk and Linder (2012) is extended to account for any chain length distribution, which will allow us to review the general constitutive equations for the nonaffine minimum averaged energy based microsphere model and to compare it to the affine model and the hybrid equal-force model within isotropic elastic assumptions. Then, in section 3, the Mullins softening is introduced with an account for directional damage and a proper treatment of the evolution of the stress-free configuration. The model, which satisfies the ClausiusDuhem inequality, is tested on the uniaxial tension stretch-stress responses of four actual materials made of the same styrene butadiene rubber gum and filled with different amounts of N347 carbon-black. Finally, a representative numerical example was chosen to illustrate the capability of the model to exhibit induced anisotropy in terms of behavior and residual stretch

\section{Elastic microsphere models with an account for molecular chain distributions}

\subsection{General equations}

Applying the thermodynamics principles to an ideal rubber network defines its free energy $W$ as,

$$
W=-T \sum_{n} s
$$

with $T$ the temperature, $s$ the entropy of a polymer chain and $n$ the number of chains. The entropy of a single non-Gaussian freely rotating chain, made of $N$ segments of lengths $b$, and characterized by its end-to-end distance $r$, 
is defined as (Kuhn and Gün, 1942),

$$
s=c(T)-k N\left(\frac{r}{N b} \beta+\ln \left(\frac{\beta}{\sinh \beta}\right)\right) \text { with } \beta=\mathcal{L}^{-1}\left(\frac{r}{N b}\right),
$$

with $c$ an arbitrary constant, $k$ the Boltzmann constant and $\mathcal{L}(x)=\operatorname{coth} x-\frac{1}{x}$ the Langevin function. Deriving Eq. (2) gives the force-elongation relation for a single chain,

$$
f=-T\left(\frac{\partial s}{\partial r}\right)=\frac{k T}{b} \mathcal{L}^{-1}\left(\frac{r}{N b}\right)
$$

More generally, one may consider chain entropy of the form

$$
s=c(T)-k N \psi\left(\frac{r}{N b}\right),
$$

leading to a force elongation law given by

$$
f=\frac{k T}{b} \psi^{\prime}\left(\frac{r}{N b}\right)
$$

the function $\psi$ being convex, and equal to zero at zero extension $(\psi(0)=0)$.

In the reference configuration, a polymer chain is characterized by its number of segments $N_{i}$ and its end-to-end vector orientation $\boldsymbol{R}(\|\boldsymbol{R}\|=1)$. In this configuration, the polymer network is described by the probability $p\left(N_{i}, \boldsymbol{R}\right)$ of encountering a chain of $N_{i}$ segments and orientation $\boldsymbol{R}$. In a microsphere representation (Figure 1), end-to-end vectors are depicted with one end at the center of the sphere and the other end at its surface. Two chains with the same initial orientation $\boldsymbol{R}$ and the same number of segments $N_{i}$ are assumed to have the same current end-to-end vector $\boldsymbol{r}=\boldsymbol{r}\left(N_{i}, \boldsymbol{R}\right)$ of norm noted $\|\boldsymbol{r}\|=r_{N_{i}, \boldsymbol{R}}$. Within this representation, the strain dependent part of the free energy density $\mathcal{W}$ becomes

$$
\mathcal{W}=\nu k T \int_{N_{i}} \iint_{S_{u}} p\left(N_{i}, \boldsymbol{R}\right) N_{i} \psi\left(\frac{r_{N_{i}, \boldsymbol{R}}}{N_{i} b}\right) d N_{i} d S_{u}
$$

with $\nu$ the number of chain per unit volume, $S_{u}$ the unit sphere and $\iint_{S_{u}} d S_{u}=$ $\frac{1}{4 \pi} \int_{\theta=0}^{\pi} \int_{\varphi=0}^{2 \pi} \sin \theta d \theta d \varphi$.

Dealing with incompressible entropic hyperelasticity, the first Piola-Kirchhoff tensor writes as,

$$
\boldsymbol{\tau}=\frac{\partial \mathcal{W}}{\partial \boldsymbol{F}}-q \boldsymbol{F}^{-T}
$$



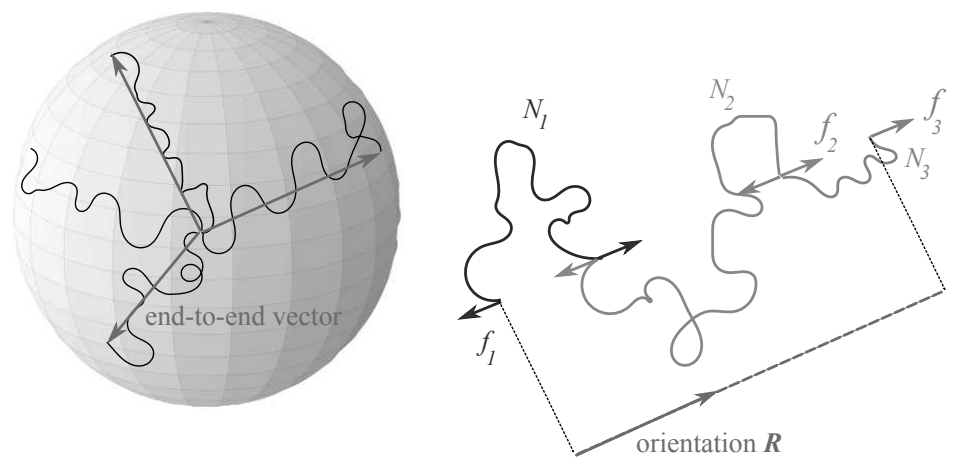

Figure 1: Microsphere approach representation with an account of chains of several lengths characterized by their number of segments $N_{i}$. The initial orientation of a chain is given by vector $\boldsymbol{R}$.

with $\boldsymbol{F}$ the macroscopic deformation gradient tensor and $q$ an unknown pressure. Note that the current constitutive equations framework could be extended to compressible materials by adding to $\mathcal{W}$ a dilatational part $U(J)$ with $J=\operatorname{det}(\boldsymbol{F})$ characterizing the volume changes. Introducing Eq. (6) in Eq. (7), one writes $\boldsymbol{\tau}$ as

$$
\boldsymbol{\tau}=\nu k T \int_{N} \iint_{S_{u}} p\left(N_{i}, \boldsymbol{R}\right) \psi^{\prime}\left(\frac{r_{\boldsymbol{R}, N_{i}}}{N_{i} b}\right) \frac{\partial r_{\boldsymbol{R}, N_{i}}}{b \partial \boldsymbol{F}} d S_{u} d N_{i}-q \boldsymbol{F}^{-T}
$$

and $\frac{\partial r_{\boldsymbol{R}, N_{i}}}{\partial \boldsymbol{F}}$ varies according to the chosen micromechanics scale transition. In what follows, we explore three existing scale changes, the affine model, the hybrid equal-force model (Verron and Gros, 2017), and the non-affine minimum energy based maximal advance path constraint (MAPC) model (Tkachuk and Linder, 2012), assuming isotropy. Within such an assumption, the probability distribution $p\left(N_{i}, \boldsymbol{R}\right)$ becomes independent of $\boldsymbol{R}$, and writes as $p\left(N_{i}\right)$.

\subsection{Affine model}

The current end-to-end vector of a chain, characterized by $N_{i}$ segments and an initial orientation $\boldsymbol{R}$, and that is part of a network submitted to the macroscopic deformation gradient $\boldsymbol{F}$, is simply defined as,

$$
\boldsymbol{r}\left(N_{i}, \boldsymbol{R}\right)=\sqrt{N_{i}} b \boldsymbol{F} \cdot \boldsymbol{R}
$$


and consequently,

$$
\frac{\partial r_{N_{i}, \boldsymbol{R}}}{\partial \boldsymbol{F}}=\frac{N_{i} b^{2}}{r_{N_{i}, \boldsymbol{R}}} \boldsymbol{F} \cdot \boldsymbol{R} \otimes \boldsymbol{R}
$$

with $\otimes$ denoting the dyadic product. For this model, note that for all $\boldsymbol{F}$, the stretching of a chain $\left(r_{N_{i}, \boldsymbol{R}} / r_{N_{i}, \boldsymbol{R}}^{0}\right.$, with $\left.\boldsymbol{r}^{0}\left(N_{i}, \boldsymbol{R}\right)=\sqrt{N_{i}} b \mathbf{1} \cdot \boldsymbol{R}\right)$, depends on its initial orientation $\boldsymbol{R}$ only. Therefore, short and long chains are equally stretched, limiting the network extensibility to the maximum extension of the shorter chains.

\subsection{Hybrid equal-force model}

The hybrid equal-force model (Verron and Gros, 2017) writes that chains with the same orientation $\boldsymbol{R}$ are submitted to the same traction force $\boldsymbol{f}_{\boldsymbol{R}}$ independently of their number of segments, which could be illustrated in Figure 1 by writing $f_{1}=f_{2}=f_{3}$. The initial equilibrated force $f^{0}$ is determined by setting the microsphere radius equal to the radius of the affine microsphere, which writes as

$$
\int_{N_{i}} p\left(N_{i}\right) N_{i} b \mathcal{L}\left(\frac{f^{0} b}{k T}\right) d N_{i}=\int_{N_{i}} p\left(N_{i}\right) \sqrt{N_{i}} d N_{i} .
$$

Moreover, the authors force the current end-to-end vector $\boldsymbol{r}$ of any chain to be aligned with the $\boldsymbol{F} \boldsymbol{R}$ for all applied $\boldsymbol{F}$. Within this constraint, the authors prove that an isotropic network characterized by the probability $p\left(N_{i}\right)$ behaves like an affine network of chains all made of $\mathcal{N}=\left(\frac{\int_{N_{i}} p\left(N_{i}\right) N_{i} d N_{i}}{\int_{N_{i}} p\left(N_{i}\right) \sqrt{N_{i}} d N_{i}}\right)^{2}$ segments of bond length $\mathcal{B}=\frac{\left(\int_{N_{i}} p(N) \sqrt{N_{i}} d N_{i}\right)^{2}}{\int_{N_{i}} p\left(N_{i}\right) N_{i} d N_{i}} b$, and characterized by the following force-elongation behavior,

$$
f=\frac{k T}{b} \mathcal{L}^{-1}\left(\frac{r}{\mathcal{N B}}\right)=\frac{k T}{b} \mathcal{L}^{-1}\left(\frac{r}{<N>b}\right) .
$$

\subsection{Non-affine mininum averaged energy model}

The non-affine microsphere minimum averaged energy based model introduced by Tkachuk and Linder (2012) was defined for a network of chains characterized by a single number of segments $N$. Upon deformation gradient $\boldsymbol{F}$, the current end-to-end lengths of chains are calculated by minimizing the 
network free energy under specific deformation constraint that the authors reduce to the following average kinematic conditions

$$
\iint_{S_{u}} \boldsymbol{r} \otimes \boldsymbol{R} d S_{u}=\frac{<r^{0}>}{3} \boldsymbol{F}
$$

with $\left\langle r^{0}\right\rangle=\sqrt{N} b$ the initial chain lengths. The constrained minimization problem is equivalent to finding a stationary point to the Lagrangian,

$$
\begin{aligned}
L(\boldsymbol{r}, \boldsymbol{\Pi}) & =k T \iint_{S_{u}} N \psi\left(\frac{r_{N, \boldsymbol{R}}}{N b}\right) d S_{u} \\
& -\boldsymbol{\Pi}:\left(\iint_{S_{u}} \boldsymbol{r} \otimes \boldsymbol{R} d S_{u}-\frac{<r^{0}>}{3} \boldsymbol{F}\right)
\end{aligned}
$$

with $\Pi$ a second-order tensor Lagrange multiplier, which was found to conveniently satisfy to (Tkachuk and Linder, 2012),

$$
f_{R}=\Pi \cdot R
$$

for every chain originally oriented along $\boldsymbol{R}$, and to,

$$
\boldsymbol{\tau}=\frac{\nu<r^{0}>}{3} \boldsymbol{\Pi}-q \boldsymbol{F}^{-T}
$$

Note that with Eq. (15), the model results into an equal-force model with chains of the same orientation $\boldsymbol{R}$ being submitted to the same traction force. Nonetheless, unlike the hybrid equal-force model, the current end-toend vectors $\boldsymbol{r}$ are not necessarily aligned with $\boldsymbol{F} \cdot \boldsymbol{R}$.

Our extension of the model to networks presenting a distribution of chain lengths rewrites the kinematic constraint as

$$
\int_{N_{i}} \iint_{S_{u}} p\left(N_{i}, \boldsymbol{R}\right) \boldsymbol{r}_{N_{i}, \boldsymbol{R}} \otimes \boldsymbol{R} d S_{u} d N_{i}=\frac{1}{3} \boldsymbol{F} \cdot \boldsymbol{l}^{0}
$$

noting

$$
\frac{1}{3} \boldsymbol{l}^{0}=\int_{N_{i}} \iint_{S_{u}} p\left(N_{i}, \boldsymbol{R}\right) \boldsymbol{r}_{N_{i}, \boldsymbol{R}}^{0} \otimes \boldsymbol{R} d S_{u} d N_{i} .
$$

For an isotropic undamaged distribution of chains, the original chain distribution will be $\boldsymbol{r}_{N_{i}}^{0}=l_{N_{i}}^{0} \boldsymbol{R}$, and from (18) the reference average chain distribution writes as

$$
\boldsymbol{l}^{0}=<r^{0}>\mathbf{1} \text { with }<r^{0}>=\int_{N_{i}} p\left(N_{i}\right) l_{N_{i}}^{0} d N_{i} .
$$


Herein, the average distance of the end-to-end chain vectors in the initial configuration $\left\langle r^{0}\right\rangle$ is chosen equal to $\int_{N_{i}} p\left(N_{i}\right) \sqrt{N_{i}} b d N_{i}$ for model comparisons. The constrained free energy minimization problem Eq. (14) reduces again to finding the stationary point of the Lagrangian

$$
\begin{aligned}
L(\boldsymbol{r}, \boldsymbol{\Pi}) & =k T \int_{N_{i}} N_{i} \iint_{S_{u}} p\left(N_{i}, \boldsymbol{R}\right) \psi\left(\frac{r_{N_{i}, \boldsymbol{R}}}{N_{i} b}\right) d S_{u} d N_{i} \\
& -\boldsymbol{\Pi}:\left(\int_{N_{i}} \iint_{S_{u}} p\left(N_{i}, \boldsymbol{R}\right) \boldsymbol{r}_{N_{i}, \boldsymbol{R}} \otimes \boldsymbol{R} d S_{u} d N_{i}-\frac{1}{3} \boldsymbol{F} \cdot \boldsymbol{l}^{0}\right) .
\end{aligned}
$$

To solve (19), the easiest way is to introduce the chain traction $f_{R}$. The optimality conditions of (19) then write

$$
\begin{aligned}
& \frac{f_{R}}{r_{N_{i}, \boldsymbol{R}}} \boldsymbol{r}_{N_{i}, \boldsymbol{R}}=\Pi \cdot \boldsymbol{R}, \\
& \int_{N_{i}} \iint_{S_{u}} p\left(N_{i}, \boldsymbol{R}\right) \boldsymbol{r}_{N_{i}, \boldsymbol{R}} \otimes \boldsymbol{R} d S_{u} d N_{i}=\frac{1}{3} \boldsymbol{F} \cdot \boldsymbol{l}^{0} .
\end{aligned}
$$

Taking the norm of (20) we get $f_{R}=\|\boldsymbol{\Pi} \cdot \boldsymbol{R}\|$, then $r_{N_{i}, \boldsymbol{R}}$ by inversion of the constitutive law $f_{R}=\frac{k T}{b} \psi^{\prime}\left(\frac{r_{N_{i}, \boldsymbol{R}}}{N_{i} b}\right)$, and after elimination of $\boldsymbol{r}_{N_{i}, \boldsymbol{R}}$ by (20), the kinematic constraint (21) reduces to an implicit nonlinear constitutive law defining the multiplier $\boldsymbol{\Pi}$ by

$$
\boldsymbol{\Pi} \cdot \int_{N_{i}} \iint_{S_{u}} p\left(N_{i}, \boldsymbol{R}\right) \frac{r_{N_{i}, \boldsymbol{R}}}{f_{R}} \boldsymbol{R} \otimes \boldsymbol{R} d S_{u} d N_{i}=\frac{1}{3} \boldsymbol{F} \cdot \boldsymbol{l}^{0} .
$$

We then get the first Piola Kirchhoff stress tensor by

$$
\begin{aligned}
\boldsymbol{\tau} & =\nu \frac{\partial L}{\partial \boldsymbol{F}}-q \boldsymbol{F}^{-T} \\
& =\frac{\nu}{3} \boldsymbol{\Pi} \cdot\left(\boldsymbol{l}^{0}\right)^{T}-q \boldsymbol{F}^{-T}
\end{aligned}
$$

Observe that by construction the second Piola Kirchhoff stress tensor $\boldsymbol{S}=$ $\boldsymbol{F}^{-1} \cdot \boldsymbol{\tau}$ is indeed symmetric since we have from $(22)$

$$
\boldsymbol{S}=\frac{\nu}{9} \boldsymbol{l}^{0} \cdot\left(\int_{N_{i}} \iint_{S_{u}} p\left(N_{i}, \boldsymbol{R}\right) \frac{r_{N_{i}, \boldsymbol{R}}}{f_{R}} \boldsymbol{R} \otimes \boldsymbol{R} d S_{u} d N_{i}\right)^{-1} \cdot\left(\boldsymbol{l}^{0}\right)^{T}-q \boldsymbol{F}^{-1} \cdot \boldsymbol{F}^{-T} .
$$




\subsection{Model comparison}

Theoretical uniaxial tension stretch-stress responses are estimated to compare the models. Within isotropy and incompressibility assumptions, the uniaxial tension deformation gradient is diagonal and depends on the uniaxial stretching $\lambda$ according to $\boldsymbol{F}=\boldsymbol{D} \boldsymbol{i a g}\left(\lambda, \lambda^{-1 / 2}, \lambda^{-1 / 2}\right)$. Using in all cases the same chain entropy (2), the engineering uniaxial stress $F / S_{0}$ is simply calculated with the previous definitions of $\boldsymbol{\tau}$ for the different models, and one obtains:

- For affine networks,

$$
F / S_{0}=\nu k T \int_{N_{i}} p\left(N_{i}\right) \iint_{S_{u}} \frac{N_{i} b}{r_{N_{i}, \boldsymbol{R}}} \mathcal{L}^{-1}\left(\frac{r_{N_{i}, \boldsymbol{R}}}{N_{i} b}\right)\left(R_{1}^{2} \lambda-\frac{R_{3}^{2}}{\lambda^{2}}\right) d S_{u} d N_{i}
$$

with $r_{N_{i}, \boldsymbol{R}}=\sqrt{N_{i}} b \sqrt{\boldsymbol{R} \cdot \boldsymbol{C} \cdot \boldsymbol{R}}$.

- For hybrid equal-force networks,

$$
F / S_{0}=\nu k T \iint_{S_{u}} \frac{\mathcal{B}}{b} \frac{\mathcal{N B}}{r_{\mathcal{N}, \boldsymbol{R}}} \mathcal{L}^{-1}\left(\frac{r_{\mathcal{N}, \boldsymbol{R}}}{\mathcal{N B}}\right)\left(R_{1}^{2} \lambda-\frac{R_{3}^{2}}{\lambda^{2}}\right) d S_{u}
$$

with $r_{\mathcal{N}, \boldsymbol{R}}=\sqrt{\mathcal{N}} \mathcal{B} \sqrt{\boldsymbol{R} \cdot \boldsymbol{C} \cdot \boldsymbol{R}}$.

- For the non-affine minimum averaged energy model,

$$
F / S_{0}=\nu \frac{<r^{0}>}{3}\left(\Pi_{11}-\frac{\Pi_{33}}{\lambda^{3 / 2}}\right)
$$

with components $\Pi_{11}$ and $\Pi_{33}$ defined by Eq. (22) that result in two nonlinear equations to solve,

$$
\left\{\begin{array}{l}
\frac{<r^{0}>}{3} \lambda-\int_{N_{i}} p\left(N_{i}\right) \iint_{S_{u}} N_{i} b \mathcal{L}\left(f_{\boldsymbol{R}} b / k T\right) \frac{\Pi_{11}}{f_{\boldsymbol{R}}} R_{1}^{2} d S_{u} d N_{i}=0 \\
\frac{<r^{0}>}{3} \frac{1}{\sqrt{\lambda}}-\int_{N_{i}} p\left(N_{i}\right) \iint_{S_{u}} N_{i} b \mathcal{L}\left(f_{\boldsymbol{R}} b / k T\right) \frac{\Pi_{33}}{f_{\boldsymbol{R}}} R_{3}^{2} d S_{u} d N_{i}=0
\end{array}\right.
$$

with $f_{\boldsymbol{R}}=\left(\sum_{i} \Pi_{i i}^{2} R_{i}^{2}\right)^{1 / 2}$ and $\Pi_{22}=\Pi_{33}$ for loading symmetry reasons.

A network made of $50 \%$ of chains with 4 segments and $50 \%$ of chains with 16 segments is chosen to compare the uniaxial stretch-stress responses of these models. Note that a simple set of 256 directions defined by Sloan and Wormersley (2004) was chosen for the numerical integration over the unit 


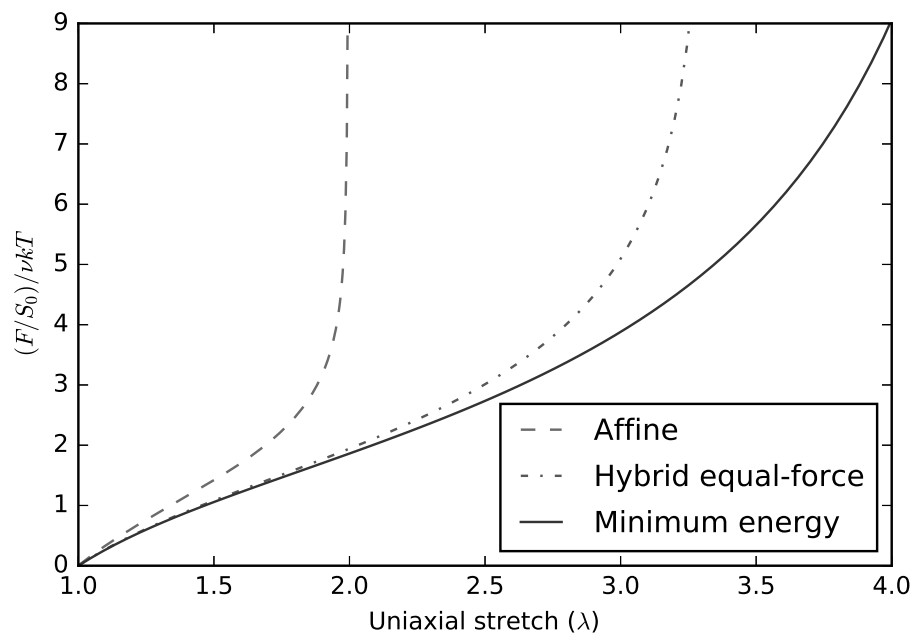

Figure 2: Normalized uniaxial tension stretch-stress responses predicted by the affine, the hybrid equal force and the minimum strain energy based models for a microsphere network characterized by $50 \%$ of chains with 4 segments and $50 \%$ of chain with $N=16$ segments.

sphere. Readers interested in optimizing the numerical integration scheme for microsphere models may refer to Ehret et al. (2010) and Verron (2015). Figure 2 shows the uniaxial stretch-stress responses, normalized by $\nu k T$, obtained with the three models. The affine model is the stiffest, and its maximum uniaxial stretch is limited by the shorter chains to $\sqrt{\min \left(N_{i}\right)}$. This property contradicts the experimental data reported for bimodal networks (Mark, 1981). The non-affine minimum averaged energy based model is the softest, with the larger limit of extension. At small and moderate stretches, the hybrid equal-force presents the same initial stiffness as the energy based model, which indicates that logically for moderate uniaxial tension the minimum energy based model displays chain end-to-end vectors that are rather aligned with the affine chain end-to-end vectors. At larger stretches, the hybrid equal-force model becomes significantly stiffer than the minimum energy based model. Actually, within the microsphere representation, the affine model presents an upper bound while the equal-force MAPC model defined with an average kinematic constraint defines a lower bound. It is no surprise that the hybrid equal-force model from Verron and Gros (2017) defined with a local kinematic constraint applied at the chain scale, appears in between the mentioned bounds. 
Before introducing damage in the model, we may discuss briefly the capability of microsphere models to reproduce rubber like materials stretch-stress responses for various states of strain. Actually, neither Tkachuk and Linder (2012) nor Verron and Gros (2017) looked into this problem. For the affine model, a satisfactory attempt to fit pure shear and biaxial stretchstress responses from data reported by James et al. (1975) may be found in (Diani and Gilormini, 2005). In the latter paper, Treloar (1944) uniaxial and equibiaxial data were fit with different values of the parameter of chain length $N$ for the fact that samples were preloaded differently according to the test, possibly inducing disparate Mullins softening. For this same reason, Treloar's data are to be used with caution when testing models. Actually, not enough information was provided by the author to use these data with confidence.

When testing the affine and the MAPC models on the reference data from Kawabata et al. (1981) recorded on an unfilled natural rubber submitted to pure shear and equibiaxial tension at stretches below the crystallization threshold, both models show unsatisfactory results Montrer figure? . This limitation may be overcome, as it was proposed by Miehe et al. (2004), by adding a tube constraint to the chain motion. The chain motion is defined within a tube of diameter $d$ and the elementary strain energy density depends on the chain stretching and on the tube contraction. The strain energy is no longer a single variable function. While Miehe et al. (2004) model provides with a much better account of the state of strain dependence of rubberlike materials, it also introduces more fitting parameters (actually 3 parameters were added). Introducing such a complexity is needed for any microsphere model, whether it is a force model or an affine model, when actual data obtained with various states of strains are considered. Nonetheless, in order to simply present a model that already presents some complication, it was chosen to use a commun form for the elementary strain energy density and to limit the model/experimental data comparisons to uniaxial tension tests.

\section{Modeling Mullins-type damage}

\subsection{Framework}

Damage such as the Mullins effect experienced by carbon-black of silica filled rubbers has been added with success to affine microsphere models (Göktepe and Miehe, 2005; Diani et al., 2006). Therefore, the non-affine 
minimum averaged energy based model is extended to account for such a directional softening generating anisotropy and resulting in the evolution of the stress-free configuration. The model will be tested on the representations of the stretch-stress responses of several carbon-black filled styrene butadiene rubbers upon cyclic uniaxial tensions. Moreover, a numerical example is presented to illustrate the model ability to produce deformation-induced anisotropy.

\subsection{Damage definition}

Extensive experimental observations have shown that at the macroscopic scale, the Mullins softening is a direction damage that is activated once exceeding the maximum stretch or equivalently the maximum load already applied in the considered direction (Merckel et al., 2012). The microsphere approach provides a convenient framework to apply directional damage, allowing for instance to modify the chain property in each direction independently. Mullins softening appears to result from the debonding and unravelling of the layer of rubber at the surface of the filler particles (Diaz et al., 2014). Therefore, links that were mechanically inactive due to restricted motions become active resulting in an increase of the number of chain links noted $N_{i}$. Consequently, the damage is implemented in the model as a directional increase of the number of chain bonds occuring at constant mass according to the loading history sustained in each direction.

Since at the chains scale, the non-affine minimum averaged energy model is an equal-force model, for each chain, the number of segments is assumed to increase with the maximum force undergone over the loading history. A simple definition of the evolution of $N_{i}$ is for instance,

$$
\left\{\begin{array}{l}
N_{i}(t)=\left[1+\alpha\left(f_{\max }-f^{i n i}\right)\right] N_{i}^{0}, f_{\max }=\max _{t}(f(t)) \text { and } \\
d N_{i} / d t=0 \text { when } f(t)<f_{\max }
\end{array}\right.
$$

where $\alpha$ is a damage parameter that is positive and constant for all directions, and $f^{i n i}$ represents the chain force in the initial stress-free isotropic condition. Since $f(t)$ depends on the chain orientation $\boldsymbol{R}$, the chain lengths evolve in a fully deterministic way unevenly according to the directions. The chain end-to-end vectors in the stress-free configuration become dependent on the chain orientation $\boldsymbol{R}$ in the reference configuration and may write as $\boldsymbol{r}_{\boldsymbol{R}, N_{i}, t}^{0}$. The stress-free configuration starts as a microsphere and then evolves with time. Figure 3 illustrates how the microsphere may evolve during a uniaxial 


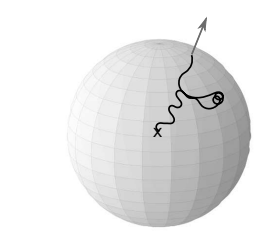

Initial configuration

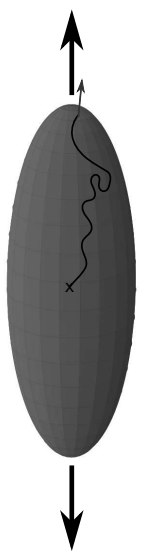

Deformed configuration undergoing damage

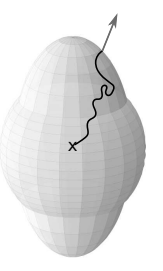

Stress-free configuration resulting from damage

Figure 3: Illustration of the possible evolution of stress-free configuration. The initial microsphere geometry is submitted to a uniaxial loading creating damage in the directions of chain extension and returns to a non-spherical stress-free configuration after unloading.

tension loading-unloading. In the next section, constitutive equations are written with the construction of the relaxed configuration. Note that in the current damage evolution, we chose to keep the probability distribution constant and we note $p_{i}=p\left(N_{i}^{0}\right)$.

\subsection{Constitutive equations with construction of the relaxed configuration}

Let us consider an initially isotropic microsphere network that has been submitted to a loading history resulting to a known distribution of numbers of segments denoted $N_{i}$ that are now dependent on $t$ and $\boldsymbol{R}$ and writes as $N_{i, \boldsymbol{R}, t}$. The polymer network is now characterized by a stress-free configuration with chain end-to-end vectors $\boldsymbol{r}_{\boldsymbol{R}, N_{i}, t}^{0}$, and when submitted to a deformation gradient tensor $\boldsymbol{F}$, the kinematic constraint Eq. (17) still holds with a stress free reference configuration given by

$$
\frac{1}{3} \boldsymbol{l}^{0}(t)=\int_{N_{i}^{0}} p_{i} \iint_{S_{u}} \boldsymbol{r}_{\boldsymbol{R}, N_{i}, t}^{0} \otimes \boldsymbol{R} d S_{u} d N_{i}^{0}
$$

and the stress tensor $\boldsymbol{\tau}$ remain defined by equations (22) and (23).

Written in a symmetric fashion, Equations (22) result again in six nonlinear equations of unknowns $\left(\boldsymbol{F}^{-1} \cdot \boldsymbol{\Pi} \cdot\left(\boldsymbol{l}^{0}\right)^{T}\right)_{i j}$, after having calculated the 
stress-free configuration $\boldsymbol{l}^{0}(t)$. To be self equilibrated, this stress free configuration must minimize the configuration free energy

$$
k T \int_{N_{i}^{0}} p_{i} \iint_{S_{u}} N_{i, \boldsymbol{R}, t} \psi\left(\frac{r_{\boldsymbol{R}, N_{i}, t}^{0}}{N_{i, \boldsymbol{R}, t} b}\right) d S_{u} d N_{i}^{0} \rightarrow \min
$$

under the volumic constraint $\operatorname{det}\left(\frac{1}{\left\langle r^{0}\right\rangle} \boldsymbol{l}^{0}\right)=1$. The optimality conditions now write

$$
\begin{aligned}
& \frac{f_{R}^{0}}{r_{\boldsymbol{R}, N_{i}, t}^{0}} \boldsymbol{r}_{\boldsymbol{R}, N_{i}, t}^{0}=\boldsymbol{\Pi}^{0} \cdot \boldsymbol{R}, \\
& \boldsymbol{\Pi}^{0}=\frac{1}{3} q^{0}\left(\boldsymbol{l}^{0}(t)\right)^{-T}, \\
& \boldsymbol{l}^{0}(t)=\frac{1}{3} \int_{N_{i}^{0}} p_{i} \iint_{S_{u}} \boldsymbol{r}_{\boldsymbol{R}, N_{i}, t}^{0} \otimes \boldsymbol{R} d S_{u} d N_{i}^{0}, \\
& \operatorname{det}\left(\frac{1}{<r^{0}>} \boldsymbol{l}^{0}\right)=1
\end{aligned}
$$

with $f_{R}^{0}=\left\|\boldsymbol{\Pi}^{0} \cdot \boldsymbol{R}\right\|$ and $r_{\boldsymbol{R}, N_{i}, t}^{0}$ given by inversion od the chain traction law $f_{R}^{0}=\frac{k T}{b} \psi^{\prime}\left(\frac{r_{\boldsymbol{R}, N_{i}, t}^{0}}{N_{i, \boldsymbol{R}, t} b}\right)$. After elimination of $\boldsymbol{l}^{0}(t)$ by (31-33), (32) reduces to a nonlinear system defining the multiplier $\boldsymbol{\Pi}^{0}$. The relaxed configuration is stress free since we have

$$
\begin{aligned}
\frac{1}{\nu} \sigma^{0}(t) & =\int_{N_{i}^{0}} p_{i} \iint_{S_{u}} \boldsymbol{f}_{R}^{0} \otimes \boldsymbol{r}_{\boldsymbol{R}, N_{i}, t}^{0} d S_{u} d N_{i}^{0}-q^{0} \mathbf{1} \\
& =\frac{1}{3} q^{0} \int_{N_{i}^{0}} p_{i} \iint_{S_{u}}\left(\boldsymbol{l}^{0}(t)\right)^{-T} \cdot \boldsymbol{R} \otimes \boldsymbol{r}_{\boldsymbol{R}, N_{i}, t}^{0} d S_{u} d N_{i}^{0}-q^{0} \mathbf{1} \\
& =0 .
\end{aligned}
$$

Note that the stress free configuration is defined within an arbitrary rotation that we set to unity by imposing that $\boldsymbol{l}^{0}(t)$ be symmetric.

\subsection{Flow rule}

In addition to the above constitutive laws, the time evolution of the internal variables $N_{i, \boldsymbol{R}, t}$ must satisfy to the Clausius-Duhem inequality for any isothermal evolution, which may writes as,

$$
-\sum_{i, \boldsymbol{R}} \frac{\partial \mathcal{W}}{\partial N_{i, \boldsymbol{R}, t}} \dot{N}_{i, \boldsymbol{R}, t} \geq 0
$$


By definition of the evolution law Eq. (28), we see that $\dot{N}_{i, \boldsymbol{R}, t}>0, \forall i$ and $\forall \boldsymbol{R}$. Moreover, deriving Eq. (6) with respect to $N_{i, \boldsymbol{R}, t}$, one obtains,

$$
\frac{\partial \mathcal{W}}{\partial N_{i, \boldsymbol{R}, t}}=\nu k T p_{i}\left(\psi\left(\frac{r}{N_{i} b}\right)-\frac{r}{N_{i} b} \psi^{\prime}\left(\frac{r}{N_{i} b}\right)\right)
$$

which is always negative since the chain strain energy function $\psi$ is convex with zero value at origin, ensuring that for each direction and each initial number of segments $N_{i}^{0}$,

$$
-\frac{\partial \mathcal{W}}{\partial N_{i, \boldsymbol{R}, t}} \dot{N}_{i, \boldsymbol{R}, t} \geq 0
$$

and therefore assessing the satisfaction of the Clausius-Duhem inequality (34).

\subsection{Reproducing experimental data}

The model is now tested to reproduce the uniaxial tension responses of four styrene butadiene rubbers made of the same gum composition and filled with 30, 40, 50 or 60 phr of N347 carbon-black. These materials were submitted to a cyclic uniaxial tests with increasing maximum stretch. Each material undergoes upon loading some significant softening that is increasing with the maximum stretch and that is exhibited by a softer response upon unloading. When reloading, the material stretch-stress response is very close to the unloading stretch-stress responses, and neglecting the material viscoelasticity, we may actually consider that the reloading and unloading stretch-stress responses coincide until reaching the maximum stretch already applied, after which the material follow the initial loading stretch-stress response. The initial loading stretch-stress responses and the unloading stretch-stress responses recorded for each materials are plotted with symbols in Figure 4.

The experimental data were fitted with the non-affine minimum energy based microsphere model accounting for Mullins softening using the microscale microstructure evolution defined in Eq. (28). For simplicity purpose, since no information was detained on the chain molecular weight distribution, only one chain length was considered noted $N^{0}$ to characterize the initial microstructure configuration. The model depends on three parameters only, $\nu k T, N^{0}$ and $\alpha$. Starting from an isotropic initial configuration, solving equations $(22,30,33)$ for an applied uniaxial stretch $\lambda$ consists in solving 4 nonlinear equations of unknown $\lambda_{t r}$ (noting $\frac{1}{<r^{0}>} \boldsymbol{l}^{0}=\boldsymbol{D i a g}\left(\lambda_{t r}, \lambda_{t r}^{-1 / 2}, \lambda_{t r}^{-1 / 2}\right)$ ), 

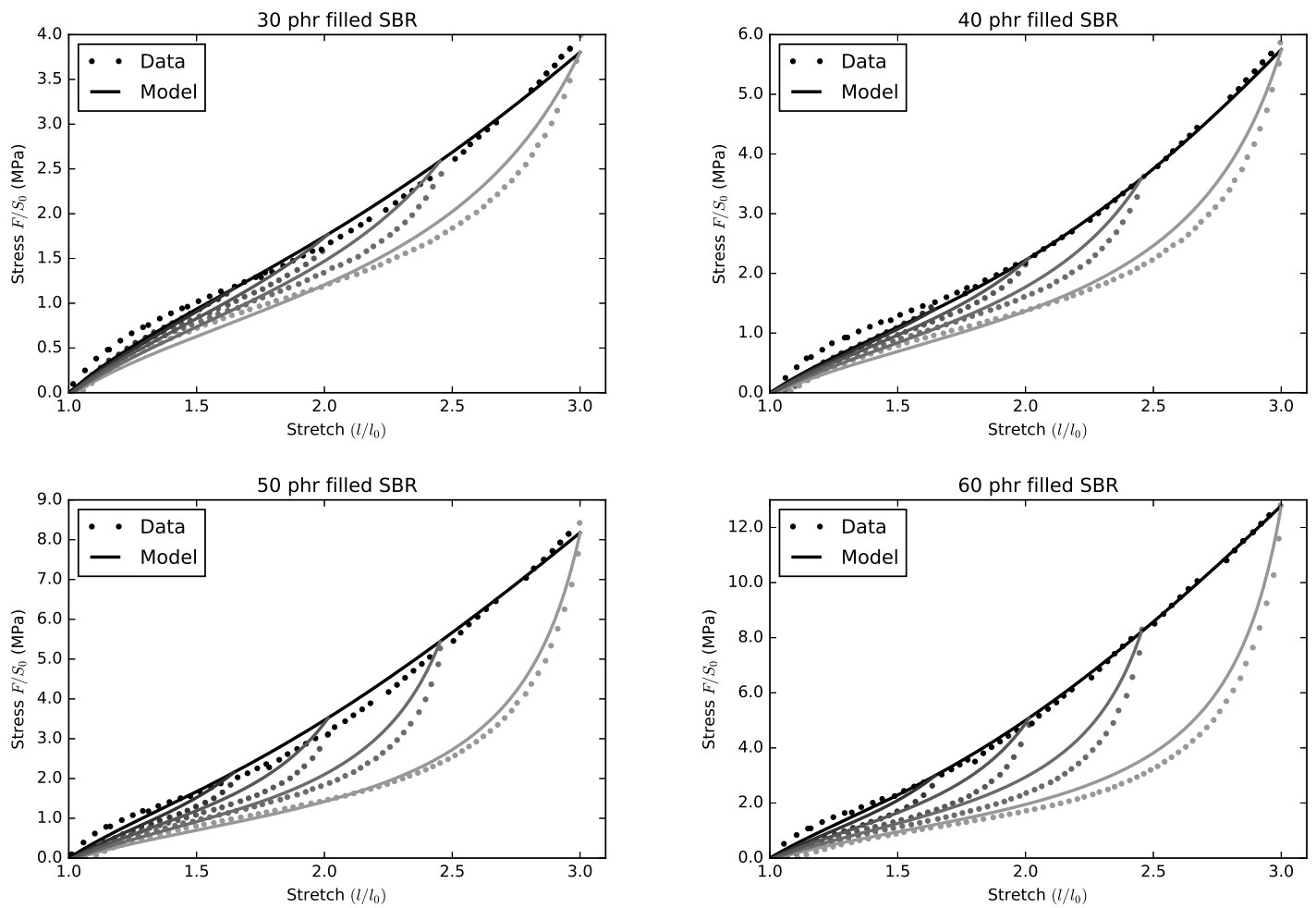

Figure 4: Model fit of the behaviors of four rubbers made of the same SBR gum and filled with either 30, 40, 50 or $60 \mathrm{phr}$ of N347 carbon-black submitted to cyclic uniaxial loading-unloadings. Experimental data are plotted with symbols and the model appear in solid lines.

$q^{0}, \Pi_{11}, \Pi_{33}$ (The algorithm used to solve the nonlinear system of equations is given in Appendix Appendix A). A fully implicit numerical scheme was successfully applied, and the representations of the model uniaxial loadingunloading responses are shown in Figure 4 for the material parameters listed in table 1. It is quite remarkable to observe that the three-parameter model reproduces well the Mullins softening for every material. Finally, one could expect a more accurate fit when the initial probability distribution $p_{i}$ is known.

\subsection{Induced anisotropy and residual stretch evolution}

As a representative numerical example to illustrate the model capability to exhibit strain-induced anisotropy, the following loading path was consid- 
Table 1: Model parameters fitted on the experimental data displayed in Figure 4.

\begin{tabular}{llll}
\hline Materials & $\nu k T(\mathrm{MPa})$ & $N^{0}$ & $\alpha$ \\
\hline 30 phr SBR & 0.63 & 3.5 & 0.080 \\
40 phr SBR & 0.67 & 3.0 & 0.047 \\
50 phr SBR & 0.75 & 2.0 & 0.054 \\
60 phr SBR & 1.0 & 2.0 & 0.042 \\
\hline
\end{tabular}

Figure 5: Model uniaxial stretch-stress responses during a first uniaxial stretching cycle according to direction 1 generating directional damage, followed by a cyclic uniaxial stretching in direction 2. The residual stretches along the principal directions of loading are plotted during the phases of loadings in order to illustrate the evolution of the stress-free configuration.

ered: First, a uniaxial tension load-unload cycle up to $200 \%$ stretching in direction 1, followed by a uniaxial tension cycle up to $200 \%$ stretching in direction 2. Figure 5 illustrates the stretch-stress responses for both cycles obtained with the $50 \mathrm{phr}$ SBR material parameters reported in table 1 . The loading response according to direction 2 is to compare to the unloading response in direction 1, which is the material response if it was submitted to a second loading according to direction 1 . The stretch-stress response difference illustrates the material anisotropy resulting from the first uniaxial cycle.

The evolution of the residual stretches during the loading steps (note that the residual stretches do not evolve during the unloadings) in the principal directions of loading are also plotted figure 5. During the first loading, the residual stretch in direction 1 increases, while the residual stretches in directions 2 and 3 are identical due to the symmetry of loading and decrease due to the material isotropy. During the subsequent stretching in direction 2 , the direction 2 residual stretch increases, while the residual stretches in directions 2 and 3 decrease non evenly due to the loss of symmetry between directions 1 and 3 induced by the first loading. 


\section{Conclusion}

The minimum energy based maximal advance path constrained model designed for microsphere representations of rubberlike materials (Tkachuk and Linder, 2012) has been extended to account for any polymer chain weight distributions and for damage such as Mullins softening experienced by filled rubbers. The equal-force model at the chain microscale, is constrained by an averaged kinematic relation at the macroscale, and appears as a lower bound for microsphere models, while the affine model assesses the upper bound.

Within the microsphere representation, chains are characterized by their reference orientation and their numbers of segments of given length. By letting the number chain segments increase, it is possible to introduce some softening resulting from enhanced chain mobility loosening the polymer network. Applying the damage on each chain independently, allows to create an uneven network consistent with the directional softening reported in the literature for the Mullins effect. Due to the uneven microstructure changes, the material initial isotropy is lost and the evolving stress-free configuration of the network has to be taken into account. A comparison between the model and actual cyclic uniaxial tension tests obtained on a SBR gum filled with four amounts of carbon-black fillers have shown the remarkable potential of the model, which reproduced relatively well the behavior of each material with three parameters only. Finally, a numerical example was proposed to illustrate the possible strain-induced anisotropy and the stress-free configuration evolution.

\section{Appendix A. Solving the constitutive equations}

Since simple uniaxial tension tests were simulated, the systems of nonlinear equations were solved with a simple python routine using the built-in fuction fsolve.

In the case of isotropy without damage, the problem consists in a system of two nonlinear equations (27). In the case of an initial isotropic material submitted to damage the problem consists in a system of five non-linear equations. Finally, when considering the numerical example illustrating the induced anisotropy, solving the second loading case consists in a system of nine non-linear equations.

- Initial state

Definition of the material parameters 
$-\nu k T$

-Damage evolution

-Definition of $n_{b}$ material directions with the chain distribution lengths yielding $<r^{0}>$

-Initialization $\boldsymbol{\Pi}=\boldsymbol{\Pi}^{0}=\frac{k T}{3<r^{0}>} \mathbf{1}$, if not first loading $\boldsymbol{\Pi}^{0}$ and $\boldsymbol{\Pi}$ are known from the previous calculations

- Loading discretization $\boldsymbol{F}\left(t_{n}\right)$

- Solving equations

-For each time step $t_{n}$, the system of equations are solved to calculate $\left(\boldsymbol{\Pi}_{t_{n}}, \boldsymbol{\Pi}_{t_{n}}^{0}, q^{0}\left(t_{n}\right)\right)$

-For every direction, calculate $\boldsymbol{f}=\boldsymbol{\Pi}_{t_{n}} \boldsymbol{R}$, update $N_{\boldsymbol{R}, t_{n}}(f)$, and

$\boldsymbol{r}=\frac{r}{f} \boldsymbol{f}$ with $r=N_{\boldsymbol{R}, t_{n}} b \mathcal{L}\left(\frac{f b}{k T}\right)$

-For every direction, calculate $\boldsymbol{f}^{0}=\boldsymbol{\Pi}_{t_{n}}^{0} \boldsymbol{R}$, and

$\boldsymbol{r}^{0}=\frac{r^{0}}{f^{0}} \boldsymbol{f}^{0}$ with $r^{0}=N_{\boldsymbol{R}, t_{n}} b \mathcal{L}\left(\frac{f^{0} b}{k T}\right)$

- Solve

$$
\begin{aligned}
& \int_{N_{i}^{0}} p_{i} \iint_{S_{u}}(\boldsymbol{r} \otimes \boldsymbol{R}) d S_{u} d N_{i}^{0}=\boldsymbol{F}\left(t_{n}\right) \cdot \int_{N_{i}^{0}} p_{i} \iint_{S_{u}}\left(\boldsymbol{r}^{0} \otimes \boldsymbol{R}\right) d S_{u} d N_{i}^{0} \\
& \boldsymbol{\Pi}^{0} \cdot\left(\int_{N_{i}^{0}} p_{i} \iint_{S_{u}}\left(\boldsymbol{r}^{0} \otimes \boldsymbol{R}\right) d S_{u} d N_{i}^{0}\right)^{T}=q^{0} \mathbf{1}, \\
& \operatorname{det}\left(\frac{1}{3<r^{0}>} \int_{N_{i}^{0}} p_{i} \iint_{S_{u}}\left(\boldsymbol{r}^{0} \otimes \boldsymbol{R}\right) d S_{u} d N_{i}^{0}\right)=1 .
\end{aligned}
$$

-Calculate the stress

$$
\boldsymbol{\tau}\left(t_{n}\right)=\nu \boldsymbol{\Pi}_{t_{n}} \cdot\left(\int_{N_{i}^{0}} p_{i} \iint_{S_{u}}\left(\boldsymbol{r}^{0} \otimes \boldsymbol{R}\right) d S_{u} d N_{i}^{0}\right)^{T}-q\left(\boldsymbol{F}\left(t_{n}\right)\right)^{-T} .
$$

\section{References}

[1] Diani, J., Brieu, M., Vacherand, J.M., 2006. A damage directional constitutive model for Mullins effect with permanent set and induced anisotropy. Eur. J. Mech. Solids A 25, 483-496.

[2] Diani, J., Gilormini, P., 2005. Combining the logarithmic strain and the full-network model for a better understanding of the hyperelastic behavior of rubber-like materials. J. Mech. Phys. Solids 53, 2579-2596. 
[3] Diaz, R., Diani, J., Gilormini, P., 2014. Physical interpretation of the Mullins softening in a carbon-black filled SBR. Polymer 55, 4942-4947.

[4] Ehret, A.E., Itskov, M., Schmide, H., 2010. Numerical integration on the sphere and its effect on the material symmetry of constitutive equations - a comparative sutdy. Int. J. Numer. Methods Eng. 81, 189-206.

[5] Göktepe, S., Miehe, C., 2005. A micro-macro approach to rubber-like materials. Part III: The micro-sphere model of anisotropic Mullins-type damage. J. Mech. Phys. Solids 53, 2259-2283.

[6] James, A.G., Green A., Simposon, G.M., 1975. Strain energy functions of rubber. ICharacterization of gum vulcanizates. J. Appl. Polymer Sci. 19, 2033-2058.

[7] Kawabata, S., Matsuda, M., Tei, K., Kawai, H., 1981. Experimental survey of the strain energy density function of isoprene rubber vulcanizate. Macromolecules, 14, 154-162.

[8] Marckmann, G., Verron, E., Gornet, L., Chagnon, G., Charrier, P., Fort, P., 2002. A theory of network alteration for the Mullins effect. J. Mech. Phys. Solids 50, 2011-2028.

[9] Mark, J.E., 1981. Rubber elasticity, Rubber Chem. Technol. 55, 11231136.

[10] Merckel, Y., Diani, J., Brieu, M., Caillard, J., 2012. A Mullins softening criterion for general loading conditions. J. Mech. Phys. Solids 60, 12571264.

[11] Miehe, C., Göktepe, S., Lulei, F., 2005. A micro-macro approach to rubber-like materials. Part I: the non-affine micro-sphere model of rubber elasticity. J. Mech. Phys. Solids 52, 2617-2660.

[12] Mullins, L., 1969. Softening of rubber by deformation. Rubber Chem. Technol. 42, 339-362.

[13] Kuhn, W., Grün, F., 1942. Beziehungen zwischen elastischen konstanten und dehnungsdoppelbrechung hochelastischer Stoffe. Kolloid-Zeitschrift 101, 248-271. 
[14] McLeish, T.C.B., 2002. Tube theory of entangled polymer dyanmics. Adv. Physics, 51, 1370-1527.

[15] Rastak, R., Linder C., 2018. A non-affine micro-macro approach to strain-crystallizing rubber-like materials. J. Mech. Phys. Solids 111, 6799.

[16] Sloan, I.H., Wormersley, R.S., 2004. Extremal systems of points and numerical integration on the sphere. Adv. Comput. Math. 21, 107-125.

[17] Tkachuk, M., Linder, C., 2012. The maximal advance path constraint for the homogenization of materials with random network microstructure. Phil. Mag. 92, 2779-2808.

[18] Treloar, L.R.G., 1944. Stress-strain data for vulcanised rubber under various types of deformation. Trans. Faraday Soc. 40, 59-70.

[19] Treloar, L.R.G., Riding, G., 1979. A non-Gaussian theory for rubber in biaxial strain? I. Mechanical properties. Proc. R. Soc. London, Ser. A 369, 261-280.

[20] Verron, E., 2015. Questioning numerical integration methods for microsphere (and microplane) constitutive equations. Mech. Mater. 89, 216228.

[21] Verron, E., Gros, A., 2017. An equal force theory for network models of soft materials with arbitrary molecular weight distribution. J. Mech. Phys. Solids 106, 176-190. 